\title{
Manejo quirúrgico de las extravasaciones por adriamicina
}

\author{
O. García Duque ${ }^{(1)}$, J. Fernández-Palacios Martínez ${ }^{(2)}$, \\ O. Cubas Sánchez ${ }^{(3)}$, F. Armas Díaz ${ }^{(4)}$ \\ (1) $Y^{(3)}$ MÉdICO INTERNO RESIDENTE. ${ }^{(2)} Y^{(4)}$ MÉDICO ADJUNTO. \\ Servicio de Cirugía Plástica y Reparadora del Hospital de Gran Canaria «DR. Negrin».
}

\author{
Correspondecia: \\ Orlando García Duque \\ Plazoleta Bagacera, 8, 2ㅇ A \\ 35016 Las Palmas de Gran Canaria \\ Tlf: 928314824 \\ e-mail: orlangd@hotmail.com
}

\begin{abstract}
Las extravasaciones de agentes quimioterápicos son complicaciones relativamente poco frecuentes, pero de gran importancia, de la terapia con estas drogas. Entre todos, la adriamicina es el agente que presenta mayor potencial ulcerógeno una vez producida la extravasación. En la actualidad el tratamiento de elección de estos accidentes es el lavado-aspiración de la zona realizado de urgencias, quedando el tratamiento quirúrgico indicado en caso que se presente necrosis cutánea o bien el dolor persista más allá de 72 horas. Las opciones quirúrgicas deben tener en cuenta no sólo el estado local de la situación sino el contexto oncológico del propio paciente. Se revisa el estado actual de la cuestión, las opciones terapéuticas y las indicaciones quirúrgicas presentando dos casos de extravasación con criterios quirúrgicos y la técnica de cobertura elegida.
\end{abstract}

Extravasations of chemotherapeutic agents are relatively rare, but very important, complications of therapy with these drugs. Out of all of them, adriamycin is the agent with the highest ulcerogenic potential once extravasation has occurred. Currently the treatment of choice is urgent cleaning-aspiration of the zone, with surgical treatment indicated in the event of cutaneous necrosis or if the pain lasts for more than 72 hours. The surgical options should take into account not only the local state of the situation but also the oncological context of the patient. We review the current state of the issue, the therapeutic options and the surgical indications by presenting two cases of extravasation with surgical criteria and the technique selected.

\section{INTRODUCCIÓN}

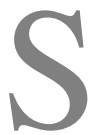
e calcula que se producen extravasaciones en el 0,1-0,7\% de los pacientes en tratamiento quimioterápico, esta incidencia se eleva al $11-58 \%$ en los niños sometidos a tratamientos intravenosos (Gault, 1993). La extravasación de determinados solutos hipertóni- cos (potásio, calcio, bicarbonato, glucosa al $10 \%$, etc.) y citotóxicos pueden originar importantes lesiones en la piel y en las estructuras subyacentes (nervios, tendones, articulaciones). Dejadas a su evolución natural el porcentaje de estas lesiones que originan necrosis cutánea se estima en un 11\% (Larson, 1985).

La adriamicina (doxorubicina hidroclorada) 


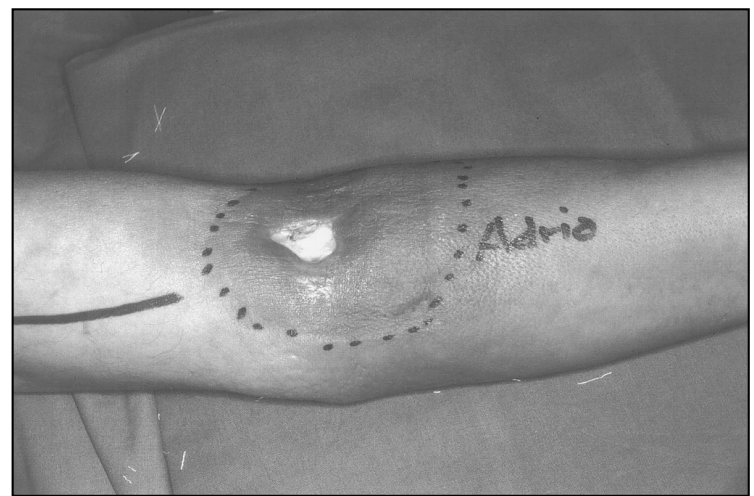

Caso 1. - Figura 1. Úlcera cutánea en flexura antecubital secundaria a extravasación por adriamicina. 30 días de evolución.

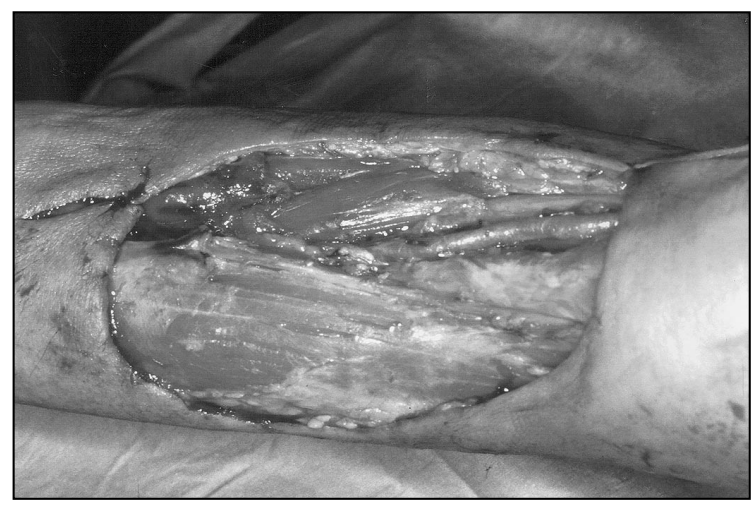

Caso 1. - Figura 2. Desbridamiento antes de la cobertura. Se puede ver la exposición de estructuras nobles profundas para cuya cobertura se precisa un colgajo.

es al agente que condiciona mayor frecuencia de necrosis cutánea tras extravasarse (Heckler, 1989). Es una antraciclina que actúa inhibiendo la síntesis de ADN y se usa clínicamente en el tratamiento de neoplasias hematológicas, sarcomas y los carcinomas de mama, pulmón y próstata. Los efectos secundarios más importantes relacionados con su empleo son la toxicidad cardiaca, la depresión de la médula ósea y la toxicidad local cuando se extravasa. Clínicamente, la extravasación de adriamicina se caracteriza por el dolor, la tumefacción y el eritema que puede acompañarse de la formación de flictenas y puede evolucionar a necrosis cutánea, que cuando se presenta suele condicionar una ulceración de evolución tórpida (Disa et al., 1998). Histológicamente, es característica la formación de complejos entre el ADN y la adriamicina que alteran la función de los miofibroblastos, lo que se traduce en una falta de contracción de la herida. Una vez muertas las células estos complejos son liberados y pueden ser endocitados por nuevas células, con lo que progresa la lesión. Este proceso, junto a la gran tendencia de esta droga a la persistencia en nuestros tejidos, se ha detectado hasta 5 meses tras el accidente (favorecido por la gran lipofilia), justifican la tendencia a la cronicidad e incluso a la progresión de estas lesiones (Heckler, 1989; Disa et al., 1998).

\section{CASOS CLÍNICOS}

\section{CASO 1:}

Paciente varón de 61 años de edad diagnóticado de mieloma múltiple Ig A Kappa estadio IIIa y en tratamiento quimioterápico con VAD + Aredia. Sufre extravasación de adriamicina a nivel de la fosa antecubital. Fuimos consultados 48 horas después del accidente. Presentaba en ese momento una placa indurada en toda la superficie de flexión del codo con una

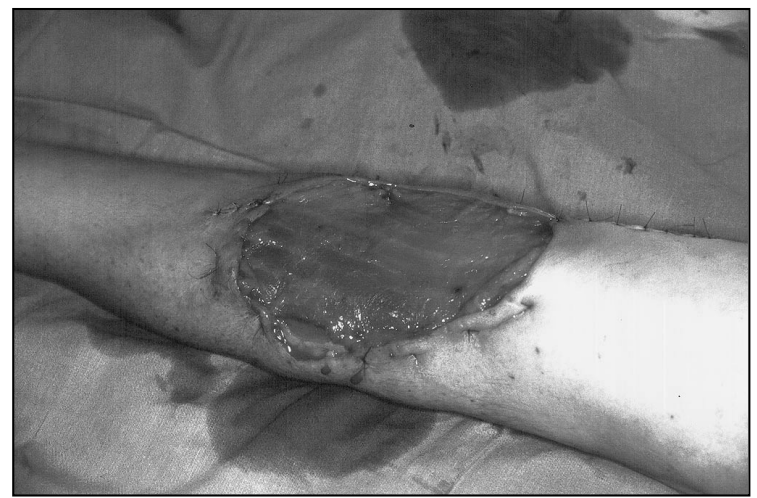

Caso 1. - Figura 3. Cobertura con colgajo muscular de latissimus dorsi pediculado.

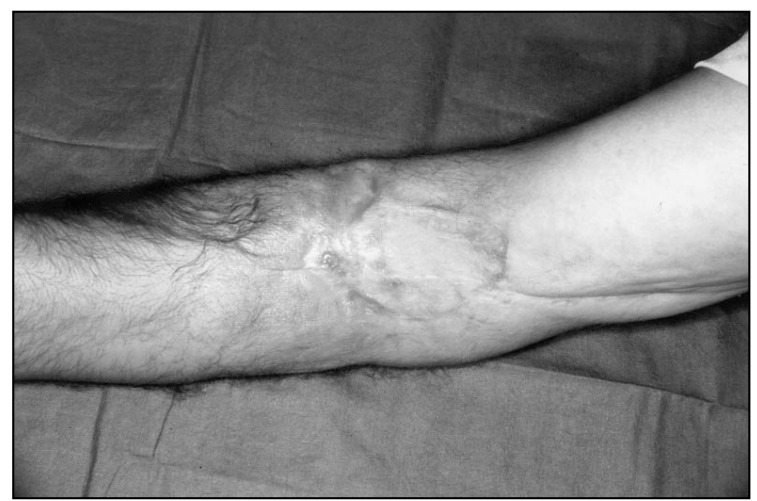

Caso 1. - Figura 4. Resultado final. Tras la toma del injerto sobre el colgajo muscular. 
escara blanquecina en la mitad de $3 \times 1 \mathrm{~cm}$. Se decidió esperar la delimitación de la necrosis ante la falta de dolor inicial del paciente. Sin embargo, tras varios días de evolución, el área de necrosis cutánea progresó y el paciente comenzó a referir un intenso dolor que no cedió tras más de 72 horas de cuidados locales. Se decide intervenir al paciente, evidenciándose internamente una gran afectación de las partes blandas de la región antecubital lo que obligó a efectuar un desbridamiento amplio de la zona quedando expuestos en profundidad los nervios radial y mediano y la arteria braquial. El defecto medía 18 x $10 \mathrm{cms}$. por lo que trascendía las posibilidades de los colgajos braquiales o antebraquiales. La cobertura se logró mediante un colgajo muscular de dorsal ancho pediculado que fue posteriormente injertado. La evolución fue satisfactoria desapareciendo el dolor y conservándose la buena función de la extremidad.

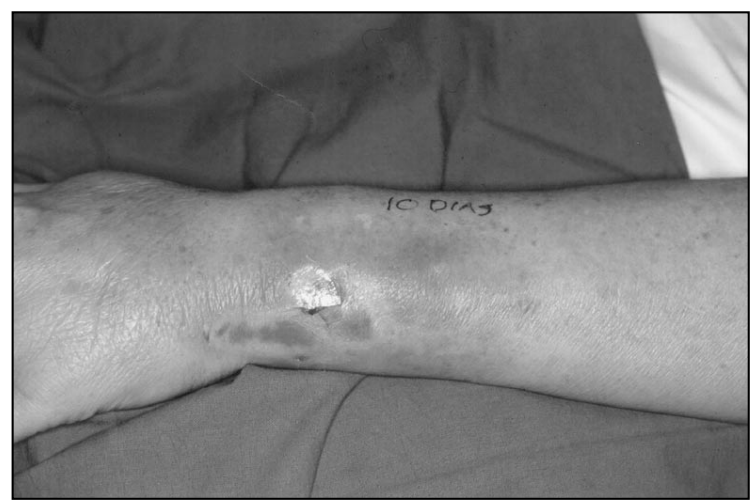

Caso 2. - Figura 1. Pérdida de sustancia en cara dorsal antebrazo secundaria a extravasación por adriamicina. 10 días de evolución.

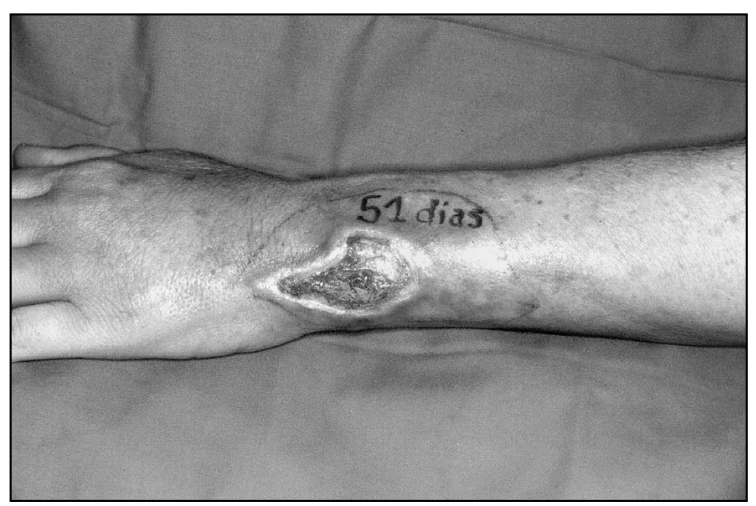

Caso 2. - Figura 2. Progresión de la lesión. 51 días de evolución.

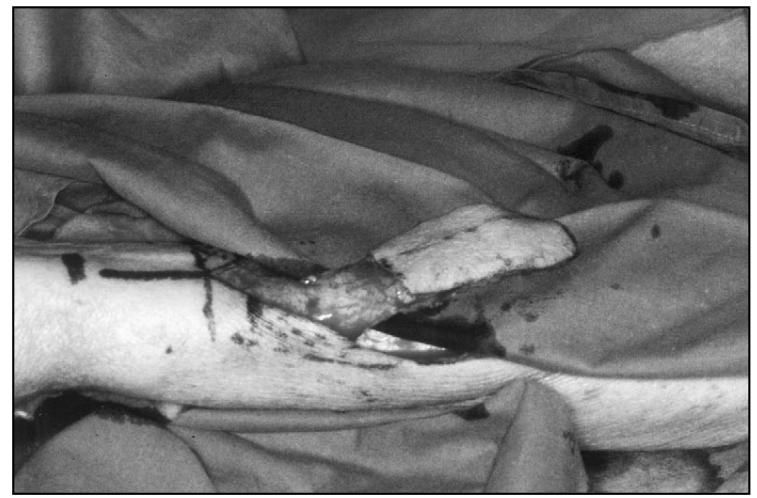

Caso 2. - Figura 3. Elevación del colgajo radial «sine arteria» basado en sus perforantes distales.

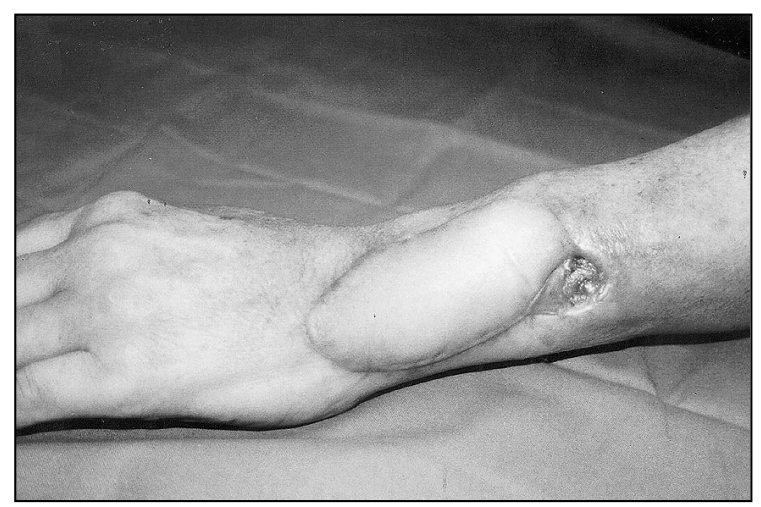

Caso 2. - Figura 4. Resultado final. Se observa la supervivencia de la totalidad del colgajo y la presencia de una pequeña área injertada, para no poner en tensión al colgajo que tardó en curar varias semanas, lo que ejemplifica la dificultad de toma que en estas lesiones tienen los injertos.

\section{CASO 2:}

Paciente mujer de 59 años de edad diagnosticada de Linfoma no Hodgkin (LNH) de bajo grado estadio IA hace 6 años siendo tratada con radioterapia, hace 3 años recibe poliquimioterapia (CHOP) por progresión de la enfermedad, hace 2 años ingresa para estudio de paraparesia diagnosticándose neoformación a nivel de D-6 que requiere extirpación y fijación columna dorsal más radioterapia. La biopsia efectuada es informada como LNH tipo B de células grandes por lo que se encuentra en tratamiento con poliquimioterapia (CHOP). En el curso de su tratamiento sufre extravasación de adriamicina en el dorso cubital de la mano izquierda siendo requerida la valoración por nuestro servicio tras más de 48 horas momento en el que ya presentaba una pequeña zona de necrosis cutánea. Se decide mantener una actitud conser- 
vadora evidenciándose la cronicidad de estas lesiones y su tendencia a la progresión, sin que se observasen datos de cicatrización espontánea tras 50 días de seguimiento. Se decide intervenir quirúrgicamente efectuándose, bajo plexo braquial, un amplio desbridamiento y cobertura mediante un colgajo en isla basado en las peforantes distales de la arteria radial. La elección de este colgajo se vio justificada por la ausencia de pulso cubital detectable con el Doppler y el empastamiento del área dorsal de la muñeca lo que impedía tanto el radial clásico como el interóseo posterior. La evolución postoperatoria fue excelente, manteniendo la paciente el miembro sin dolor y activo.

\section{DISCUSIÓN}

Clásicamente, no ha existido consenso en cuanto a cuál era la mejor estrategia terapéutica en este tipo de lesiones. Algunos cirujanos defendían un tratamiento radical, animados por los estudios clínicos e histológicos que evidenciaban la tendencia a la cronicidad y a la progresión de estas lesiones (Heckler, 1989). Otros defendían una actitud expectante (Larson, 1985) justificada por la baja proporción de pacientes con extravasaciones que, tratados con terapias conservadoras, acabarían desarrollando necrosis cutánea.

En la actualidad, el tratamiento de elección mantiene una aproximación intermedia que aprovecha las ventajas de la actitud radical, con la eliminación precoz del soluto extravasado, conservando al mismo tiempo la cubierta cutánea. Este tratamiento debe efectuarse de urgencias, marcando como límite las primeras 24 horas, y consiste en la combinación del lavado con suero fisiológico hialuronidasa o condroetilsulfatasa (Gil y Mateu, 1998) y la lipoaspiración del panículo subcutáneo de la zona afecta. Esta técnica de lavado-aspiración ha demostrado excelentes resultados tanto en estudios de laboratorio como en la práctica clínica y se fundamenta en los trabajos originales de Gault (Gault, 1993; Lambert y Couturaus y Arnaud, 2000; Martín y Carver y Petros, 1994; Scuderi y Onesti, 1994; Vandeweyer y Heymans y Deraemaecker, 2000).
Siguiendo este enfoque, el tratamiento quirúrgico clásico quedaría restringido a las extravasaciones remitidas tardíamente que presentan necrosis cutáneas ya establecidas y a aquellas en que persista el dolor pasadas 72 horas desde el accidente (Lambert y Couturaud y Arnaud, 2000; Scuderi y Onesti, 1994). Las técnicas quirúrgicas que se emplearán, en estos casos, dependerán del sitio de extravasación y de la importancia del daño, debiendo tenerse en cuenta las especiales circunstancias personales de estos pacientes y de su enfermedad de base. El hecho de tratarse, además, de una lesión yatrogénica, ayuda poco en la relación entre el paciente y su cirujano. En general, las lesiones producidas serán profundas dejando expuestas estructuras nobles por lo que precisarán cobertura con colgajos, que serán de vecindad, debido a que las intervenciones de mayor envergadura estarían contraindicadas en estos pacientes. El desbridamiento debe partir siempre desde tejido sano con anatomía no afecta con vistas a la identificación de los elementos nobles a preservar. La mayoría de los accidentes se producen afortunadamente en las extremidades superiores, donde existe gran cantidad de colgajos pediculados disponibles. Conviene, sin embargo, antes de decidirnos por alguno de ellos, el hacer una correcta evaluación de la situación, teniendo en cuenta el fenómeno «iceberg» de la lesión, donde las estructuras profundas están más dañadas que lo que la úlcera y su halo inflamatorio sugieren. En el segundo caso presentado, la interrupción de la arteria cubital y el empastamiento de la cara dorsal de la muñeca -área de conexión entre ambos paquetes interóseos- precluían el uso de colgajos arteriales fasciocutáneos tipo C — radial o cubital— y del interóseo posterior. Por otro lado, la utilización de colgajos locales, cuando es posible, restringe el campo quirúrgico a la extremidad afecta, por lo que se puede realizar la cirugía bajo anestesia locorregional - plexo braquial - obteniéndose una ventaja añadida en estos pacientes. La utilización de injertos es posible cuando el lecho sea adecuado (Heckler, 1989), siendo además un acto quirúrgico sencillo y rápido. Sin embargo, las zonas de venopunción más frecuentes - dorso de mano, muñeca o flexura de codo- no son proclives a presentarlo al ser zonas de escaso tejido 
subdérmico, con muchos elementos tendinosos y pocos musculares como corresponde a las zonas de transición articular.

En conclusión, nuestra misión como cirujanos ante las extravasaciones pasa en primer lugar por informar a los Servicios remitentes de pacientes - Hematología, Oncología... - sobre la importancia que tiene la premura de la remisión, para poder instaurar un tratamiento quirúrgico «conservador» a base de dilución y as- piración con cánula. Si ese tiempo ha sido ya sobrepasado se impone un control de la herida, sentando la evolución y la sintomatología la indicación quirúrgica. Por último, conviene realizar una evaluación detenida tanto de la lesión como del estado general del paciente, con vistas a las opciones quirúrgicas, siendo lo teóricamente ideal una intervención corta, bajo anestesia locorregional y utilizando un colgajo local como cobertura.

\section{BIBLIOGRAFÍA}

Disa, J. J.; Chang, R. R.; Mucci, S. J.; Goldberg, N. H.: Prevention of Adriamicin-induced fullthickness skin loss using hyaluronidase infiltration. Plast Reconstr Surg. 1998 Feb; 101 (2): 370-4.

Gault, D. T.: Extravasation injuries. Br J Plast Surg. 46: 91,1993.

Gil, M. E.; Mateu, J.: Treatment of extravasation from parenteral nutrition solution. Ann Pharmacother. 1998 Jan; 32 (1): 51-5.

Heckler, F. R.: Current thoughts on extravasation injuries. Clinics in Plastic Surgery. 1989; 16 (3): 557-63.
Lambert, F.; Couturaud, B.; Arnaud, E.: Extravasaciones yatrogénicas de solutos citotóxicos 0 hiperosmolares. Tratamiento. Enciclopedia medico-quirúrgica: cirugía plástica, reparadora y estética. Ed: Elseiwer. 2000. Cap 45-146. Pp: 1-5.

Larson, D. L.: What is the appropiate management of tissue extravasation by antitumor agent? Plas Reconstr Surg. 1985, 75: 397.

Martin, P. H.; Carver, N.; Petros, J.: Use of liposuction and saline washout for the treatment of extensive subcutaneus extra- vasation of corrosive drugs. $\mathrm{Br}$ J. Anaesth. 1994; 72: 702-4

Scuderi, N.; Onesti, M. G.: Antitumor agents: extravasation, management, and surgical treatment. Ann Plast Surg. 1994 Jan; 32 (1): 39-44.

Seng-Feng, J.; Fu-Chan, W.: The distally based forearm island flap in hand reconstruction. Plas Reconst Surg. Aug; 102 (2): 400-6.

Vandeweyer, E.; Heymans, O.; Deraemaecker, R.: Extravasation injuries and emergency suction as treatment. Plast Reconstr Surg. 2000 Jan; 105 (1): 109110. 